Article

\title{
The Mathematical Model and Numerical Solution of Instantaneous Availabilities for the Two-Unit Series Repairable System and Parallel Repairable System with Three States
}

\author{
Yi Yang ${ }^{1, *(\mathbb{D},}$, Qianbin $\mathrm{Li}^{2}$ and Xuefeng Chen ${ }^{2} \mathbb{D}$ \\ 1 School of Reliability and System Engineering, Beihang University, Beijing 100191, China \\ 2 School of Science, Nanjing University of Science and Technology, Nanjing 210094, China; \\ n958505669@163.com (Q.L.); cgndxmg412@njust.edu.cn (X.C.) \\ * Correspondence: yang_cissy@163.com
}

Received: 31 August 2018; Accepted: 17 September 2018; Published: 29 September 2018

\begin{abstract}
This paper is aimed to obtain the instantaneous availabilities (IAs) for the two-unit series system and parallel system with three states. By the compound Simpson formula and the compound trapezoidal formula, we get the numerical solution of IA for the two-unit series system with three states based on the renewal process. With four-order Runge-Kutta formula, the numerical solution of IA for the two-unit parallel system with three states is obtained based on the Markov process.
\end{abstract}

Keywords: instantaneous availability; the numerical expression; the series system; the parallel system; three states

\section{Introduction}

Nowadays, availability theory plays a more and more important role in many fields, such as information network [1], military field [2], traffic field [3] and some other basic fields [4,5]. Steady-state availability as an important aspect of reliability theory, which representing the proportion of time in normal state after a long run, has been studied for many years [6-8]. However, with the rapid development of science and technology, equipment updating and out becomes faster and the equipment service period may now be only a few years or less. Moreover, the complicated mechatronic systems nowadays always consist of a lot of mechanical elements. The interaction of subsystems often leads to undulations of the availability in the early use of new equipment. Therefore, the availability of these complicated systems usually fluctuates heavily in the early stage, but this fluctuation is quite far from the steady-state availability to be adopted for a long period by system developers and users. This factor seriously affects their understanding of the system availability level and the evaluation of the system performance of these complicated mechatronic systems.

Under this circumstance, the research on the instantaneous availability (IA) of complicated mechatronic system starts to receive wide attention $[9,10]$. IA represents the probability that the system is working at any point of time. With IA, we can easily know the fluctuation of availability of a system and evaluate the performance of a system. Currently, the mathematical analysis on the IA fluctuation of complicated mechatronic systems is unfolded from two main aspects. The first one is a study of how to set up an IA model, solve the IA and analyze the IA fluctuation for one-unit system with multiple states (working, delay-repair, repair, etc.) [11-13]. Traditionally, the most popular method to assess IA has been the 2-state (working-repair) model [11]. In such case that the system failure time and repair time follow exponential distribution, Markov process is widely used [14]. If they follow general distribution (e.g., uniform, gamma, and Weibull distributions), the Markov renewal process 
and simulation analysis are often used [11]. Yang et al. studied the fluctuation mechanism of 3-state IA model through simulation analysis and concluded that when parameters, including the failure time, the maintenance time and the support delay time, follow exponential distribution, IA of system has no fluctuation $[15,16]$. The second aspect is a study of how to set up an IA model, solve the IA and analyze the IA fluctuation for multi-unit systems (series system and parallel system). Based on the renewal model, the IA fluctuation of the two-unit series repairable system is analyzed in [17]. It turns out that there exists no fluctuation of IA if the working time and repair time are under exponential distribution.

However, among all the literatures which we can get hold of, none of them combines these two aspects together, i.e., studies how to set up an IA model, solve the IA and analyze the IA fluctuation for multi-unit series repairable systems or parallel repairable systems with multiple states. So, in this paper, we will analyze the mathematical model and IA fluctuations for the two-unit series repairable system and parallel repairable system with three states. Firstly we will give the mathematical models of the IAs of the series systems and the parallel systems with three states. Then the numerical solutions of IAs will be given to analyze the fluctuations of IAs. Finally, some numerical examples will be given to show the effectiveness of the methods in this paper.

This paper is organized as follows. In Section 2, we study the numerical solution of IA for two-unit series system with three states. Section 3 shows the numerical solution of IA for two-unit parallel system with three states. Section 4 concludes this paper.

\section{IA for Two-Unit Series System with Three States}

In this section we will establish the renewal model of IA for the two-unit series system with three states and study the numerical solution.

\subsection{Mathematical Model of IA}

For establishing the renewal model of IA for the two-unit series system with three states, the system must meet the following assumptions.

Assumption 1. Each unit $i,(i=1,2)$ has the working time $X_{i}$, the delay-repair time $W_{i}$ and the repair time $Y_{i}$, which are subject to the following exponential functions:

$$
\begin{aligned}
& X_{i} \sim F_{i}(t)=1-e^{-\lambda_{i} t}, \\
& W_{i} \sim W_{i}(t)=1-e^{-\mu_{i} t}, \\
& Y_{i} \sim G_{i}(t)=1-e^{-v_{i} t},
\end{aligned}
$$

where $\lambda_{i}$ is the fault rate, $\mu_{i}$ delay-repair rate and $v_{i}$ repair rate.

Note that the electronic units are always interfered by internal or external factors and easily lead to various types of failure, which always is the main reason for the failure of mechatronic system. The lifetime of the units generally follows exponential distribution [18]. Moreover, the maintenance time of system is generally under exponential, Weibull, logarithmic normal distribution [19]. For simplification, assume that repair time and delay-repair time are under exponential distribution.

Assumption 2. We suppose that all units are new at the beginning. Besides, the unit will be new after maintenance.

According to the assumptions above, the renewal process of the two-unit series system with three states is established by the failure time $X_{1}, X_{2}$, the delay-repair time $W_{1}, W_{2}$ and the repair time $Y_{1}, Y_{2}$ and is shown in Figure 1. $\left\{\xi_{k}+\varphi_{k}+\chi_{k}, k=1,2, \ldots, n\right\}$ is the renewal process. $\xi_{k}$ is the failure time of the series system, which means $\xi_{k}=\min X_{i}$. The delay-repair time $\varphi_{k}$ and the repair time $\chi_{k}$ rely on $\xi_{k}$, which means that $\varphi_{k}=W_{i}, \chi_{k}=Y_{i}$ when $\xi_{k}=X_{i}, i=1,2$. 


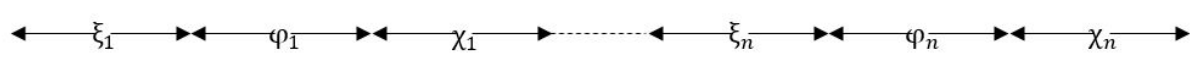

Figure 1. The renewal process of the two-unit series system with three states.

Based on the renewal process, we can obtain IA of the two-unit series system with three states as follows:

$$
\begin{aligned}
A(t)= & P(X(t)=1 \mid \text { the system is new at the beginning }) \\
= & P\left(X(t)=1, \xi_{1}>t \mid \text { the system is new at the beginning }\right) \\
& +P\left(X(t)=1, \xi_{1} \leq t<\xi_{1}+\varphi_{1}+\chi_{1} \mid\right. \text { the system is new at the beginning) } \\
& +P\left(X(t)=1, \xi_{1}+\varphi_{1}+\chi_{1} \leq t \mid \text { the system is new at the beginning }\right) .
\end{aligned}
$$

From (4) we can know that $A(t)$ consists of three parts:

The first part is

$$
\begin{aligned}
& P\left(X(t)=1, \xi_{1}>t \mid \text { the system is new at the beginning }\right) \\
= & 1-P\left(\xi_{1} \leq t \mid \text { the system is new at the beginning }\right) \\
= & 1-\left(P\left(X_{1} \leq X_{2}, X_{1} \leq t \mid \text { the system is new at the beginning }\right)\right. \\
& \left.+P\left(X_{2} \leq X_{1}, X_{2} \leq t \mid \text { the system is new at the beginning }\right)\right) \\
= & 1-\left(\int_{0}^{t} e^{-\lambda_{2} u} \lambda_{1} e^{-\lambda_{1} u} d u+\int_{0}^{t} e^{-\lambda_{1} v} \lambda_{2} e^{-\lambda_{2} v} d v\right) \\
= & e^{-\left(\lambda_{1}+\lambda_{2}\right) t} .
\end{aligned}
$$

The second part is

$$
P\left(X(t)=1, \xi_{1} \leq t<\xi_{1}+\varphi_{1}+\chi_{1} \mid \text { the system is new at the beginning }\right)=0 .
$$

The third part is

$P\left(X(t)=1, \xi_{1}+\varphi_{1}+\chi_{1} \leq t \mid\right.$ the system is new at the beginning $)$

$=\int_{0}^{t} P\left(X(t)=1 \mid\right.$ the system is new at the beginning, $\left.\xi_{1}+\varphi_{1}+\chi_{1}=u\right) d P\left(\xi_{1}+\varphi_{1}+\chi_{1} \leq u\right)$

$=\int_{0}^{t} P(X(t)=1 \mid$ the system is new at time $\mathrm{u}) d P\left(\xi_{1}+\varphi_{1}+\chi_{1} \leq u\right)$

$=\int_{0}^{t} P(X(t-u)=1 \mid$ the system is new at the beginning $) P^{\prime}\left(\xi_{1}+\varphi_{1}+\chi_{1} \leq u\right) d u$

Let $Q(u)=P\left(\xi_{1}+\varphi_{1}+\chi_{1} \leq u\right)$, so the renewal equation is as follows:

$$
A(t)=e^{-\left(\lambda_{1}+\lambda_{2}\right) t}+\int_{0}^{t} A(t-u) Q^{\prime}(u) d u .
$$

For $Q^{\prime}(t)$ in (8), we have

$$
\begin{aligned}
Q^{\prime}(t) & =P^{\prime}\left(\xi_{1}+\varphi_{1}+\chi_{1} \leq t\right)=P\left(\xi_{1}+\varphi_{1}+\chi_{1}=t\right) \\
& =P\left(X_{1} \leq X_{2}, X_{1}+W_{1}+Y_{1}=t\right)+P\left(X_{2} \leq X_{1}, X_{2}+W_{2}+Y_{2}=t\right) \\
& =\int_{0}^{t} e^{-\lambda_{2} u} P^{\prime}\left(W_{1}+Y_{1} \leq t-u\right) \lambda_{1} e^{-\lambda_{1} u} d u+\int_{0}^{t} e^{-\lambda_{1} u} P^{\prime}\left(W_{2}+Y_{2} \leq t-u\right) \lambda_{2} e^{-\lambda_{2} u} d u \\
& =\lambda_{1} \int_{0}^{t} P^{\prime}\left(W_{1}+Y_{1} \leq t-u\right) e^{-\left(\lambda_{1}+\lambda_{2}\right) u} d u+\lambda_{2} \int_{0}^{t} P^{\prime}\left(W_{2}+Y_{2} \leq t-u\right) e^{-\left(\lambda_{1}+\lambda_{2}\right) u} d u .
\end{aligned}
$$


Meanwhile we have

$$
\begin{aligned}
& P^{\prime}\left(W_{1}+Y_{1} \leq t\right)=P\left(W_{1}+Y_{1}=t\right)=\int_{0}^{t} u_{1} e^{-u_{1}(t-u)} v_{1} e^{-v_{1} u} d u=\frac{\mu_{1} v_{1}}{v_{1}-\mu_{1}}\left(e^{-\mu_{1} t}-e^{-v_{1} t}\right), \\
& P^{\prime}\left(W_{2}+Y_{2} \leq t\right)=P\left(W_{2}+Y_{2}=t\right)=\int_{0}^{t} u_{2} e^{-u_{2}(t-u)} v_{2} e^{-v_{2} u} d u=\frac{\mu_{2} v_{2}}{v_{2}-\mu_{2}}\left(e^{-\mu_{2} t}-e^{-v_{2} t}\right) .
\end{aligned}
$$

\subsection{Numerical Solution and Error Analysis}

When the failure time $X_{i}$, the delay-repair time $W_{i}$ and the repair time $Y_{i}$ obey ordinary exponential distribution, it is difficult to get the analytic expression of $A(t)$, so we will use numerical method to obtain $A(t)$. In the following, we will introduce some definitions and lemmas.

Definition 1 ([20]). Divide the interval $[a, b]$ into $n$ equivalent. Let $x_{k}=a+k h, h=\frac{b-a}{n}(k=0,1, \ldots, n)$, then apply trapezoidal formula on $f(x)$ in the interval $\left[x_{k}, x_{k}+1\right],(k=0,1, \ldots, n-1)$

$$
\begin{aligned}
I & =\int_{a}^{b} f(x) d x=\sum_{k=0}^{n-1} \int_{x_{k}}^{x_{k+1}} f(x) d x \approx \sum_{k=0}^{n-1} \frac{h}{2}\left[f\left(x_{k}\right)+f\left(x_{k+1}\right)\right]+R_{n}(f) \\
& =T_{n}+R_{n}(f),
\end{aligned}
$$

where $R_{n}(f)$ is reminder and

$$
T_{n}=\frac{h}{2}\left[f(a)+2 \sum_{k=0}^{n-1} f\left(x_{k}\right)+f(b)\right]
$$

is called the compound trapezoidal formula.

Lemma 1 ([20]). The reminder of the compound trapezoidal formula is

$$
R_{n}(f)=-\frac{b-a}{12} h^{2} f^{\prime \prime}(\eta)
$$

which means the error of compound trapezoidal formula is $O\left(h^{2}\right)$.

Definition 2 ([20]). Divide the interval $[a, b]$ into n equivalent. Let $x_{k}=a+k h, h=\frac{b-a}{n}(k=0,1, \ldots, n)$, then apply Simpson formula to $f(x)$ in the interval $\left[x_{k}, x_{k}+1\right],(k=0,1, \ldots, n-1)$

$$
\begin{aligned}
I & =\int_{a}^{b} f(x) d x=\sum_{k=0}^{n-1} \int_{x_{k}}^{x_{k+1}} f(x) d x \approx \sum_{k=0}^{n-1} \frac{h}{6}\left[f\left(x_{k}\right)+4 f\left(\frac{x_{k}+x_{k+1}}{2}\right)+f\left(x_{k+1}\right)\right]+R_{n}(f) \\
& =S_{n}+R_{n}(f),
\end{aligned}
$$

where $R_{n}(f)$ is reminder and

$$
S_{n}=\frac{h}{6}\left[f(a)+4 \sum_{k=0}^{n-1} f\left(\frac{x_{k}+x_{k+1}}{2}\right)+\sum_{k=1}^{n-1} f\left(x_{k}\right)+f(b)\right]
$$

is called the compound Simpson formula.

Lemma 2 ([20]). The reminder of the compound Simpson formula is

$$
R_{n}(f)=-\frac{b-a}{2880} h^{4} f^{(4)}(\eta)
$$


which means that the error of the compound Simpson formula is $O\left(h^{4}\right)$.

Lemma 3 (discrete Gronwall inequation [21]). If discrete series $\left\{w_{n}: n=0, \ldots, N\right\}$ satisfy

$$
w_{n} \leq A+h \sum_{k=1}^{n} B_{k} w_{k}, n=0, \ldots, N
$$

where $A, B_{k}(k=1, \ldots, N)$ are nonnegative constant; $h=\frac{1}{N}$ and $h \underset{1 \leq k \leq N}{\max }\left(B_{k}\right) \leq \frac{1}{2}$. Then

$$
\max _{0 \leq n \leq N}\left|w_{n}\right| \leq A \cdot \mathrm{e}^{2 h \sum_{k=1}^{N} B_{k}} .
$$

Next, according to the definitions and lemmas above, we will obtain $A(t)$ for the two-unit series system with three states. For obtaining $A(t)$, we will apply the compound Simpson formula and compound trapezoidal to the two integrals in the renewal Equations (8)-(11) respectively to transport the renewal equation into discrete equations. The specific steps are as follows:

STEP 1: Apply the compound Simpson formula to $Q^{\prime}(t)$. Divide the interval $[0, T]$ into $n$ equivalent. Let $0=t_{0}<t_{1}<\ldots<t_{n-1}<t_{n}=t, h=\frac{T}{n}$, then apply compound Simpson formula to $Q^{\prime}(t)$.

STEP 2: Apply the compound trapezoidal formula to $\int_{0}^{t_{i}} A(u) Q^{\prime}\left(t_{i}-u\right) d u$. Same as the division in STEP 1, we apply the compound trapezoidal formula to $\int_{0}^{t_{i}} A(u) Q^{\prime}\left(t_{i}-u\right) d u$, therefore we can obtain

$$
\int_{0}^{t_{i}} A(u) Q^{\prime}(t-u) d u \approx \frac{h}{2}\left[A\left(t_{0}\right) Q^{\prime}\left(t_{i}-t_{0}\right)+2 \sum_{k=1}^{i-1} A\left(t_{k}\right) Q^{\prime}\left(t_{i}-t_{k}\right)+A\left(t_{i}\right) Q^{\prime}\left(t_{i}-t_{i}\right)\right] .
$$

STEP 3: Bring $\int_{0}^{t_{i}} A(u) Q^{\prime}\left(t_{i}-u\right) d u$ into $A(t)=e^{-\left(\lambda_{1}+\lambda_{2}\right) t}+\int_{0}^{t} A(u) Q^{\prime}(t-u) d u$, then we can get

$$
A\left(t_{i}\right) \approx \tilde{A}\left(t_{i}\right)=F\left(t_{i}\right)+\frac{h}{2}\left[A\left(t_{0}\right) Q^{\prime}\left(t_{i-0}\right)+2 \sum_{k=1}^{i-1} A\left(t_{k}\right) Q^{\prime}\left(t_{i-k}\right)+A\left(t_{i}\right) Q^{\prime}\left(t_{i-i}\right)\right] .
$$

Then (21) can be rewritten as follows

$$
\left(I-\frac{h}{2} \tilde{Q}\right) \tilde{A}=F,
$$

where

$$
\tilde{A}=\left[\begin{array}{c}
\tilde{A}\left(t_{0}\right) \\
\tilde{A}\left(t_{1}\right) \\
\tilde{A}\left(t_{2}\right) \\
\vdots \\
\tilde{A}\left(t_{0}\right)
\end{array}\right], F=\left[\begin{array}{c}
F\left(t_{0}\right) \\
F\left(t_{1}\right) \\
F\left(t_{2}\right) \\
\vdots \\
F\left(t_{n}\right)
\end{array}\right], \tilde{Q}=\left[\begin{array}{ccccc}
0 & & & & \\
Q^{\prime}\left(t_{1}\right) & Q^{\prime}\left(t_{0}\right) & & & \\
Q^{\prime}\left(t_{2}\right) & 2 Q^{\prime}\left(t_{1}\right) & Q^{\prime}\left(t_{0}\right) & & \\
\vdots & & & & \\
Q^{\prime}\left(t_{n}\right) & 2 Q^{\prime}\left(t_{n-1}\right) & \cdots & 2 Q^{\prime}\left(t_{1}\right) & Q^{\prime}\left(t_{0}\right)
\end{array}\right]
$$

According to (22), we can obtain the approximate value $A\left(t_{i}\right)$, and thus we can get the numerical solution of $A(t)$. 


\section{Error Analysis}

On one hand, according to the steps above and combined with Definition 2, Equation (8) can be rewritten as follows:

$$
A\left(t_{i}\right)=e^{-\left(\lambda_{1}+\lambda_{2}\right) t_{i}}+\frac{h}{2}\left[A\left(t_{0}\right) Q^{\prime}\left(t_{i}-t_{0}\right)+2 \sum_{k=1}^{i-1} A\left(t_{k}\right) Q^{\prime}\left(t_{i}-t_{k}\right)+A\left(t_{i}\right) Q^{\prime}\left(t_{i}-t_{i}\right)\right]+O\left(h^{2}\right) .
$$

Then based on Lemma 2, the error of the compound Simpson formula is $O\left(h^{4}\right)$, thus

$$
Q^{\prime}(t)=\tilde{Q}^{\prime}(t)+O\left(h^{4}\right)
$$

where $\tilde{Q}^{\prime}(t)$ is the approximate value of $Q^{\prime}(t)$. Bring (25) into (24), then we can obtain

$$
A\left(t_{i}\right)=e^{-\left(\lambda_{1}+\lambda_{2}\right) t_{i}}+\frac{h}{2}\left[A\left(t_{0}\right)\left(\tilde{Q}^{\prime}\left(t_{i}-t_{0}\right)+O\left(h^{4}\right)\right)+2 \sum_{k=1}^{i-1} A\left(t_{k}\right)\left(\tilde{Q}^{\prime}\left(t_{i}-t_{k}\right)+O\left(h^{4}\right)\right)+O\left(h^{2}\right) .\right.
$$

On the other hand, the expression of $\widetilde{A}\left(t_{i}\right)$ which is the approximate value of $A\left(t_{i}\right)$ is

$$
\widetilde{A}\left(t_{i}\right)=e^{-\left(\lambda_{1}+\lambda_{2}\right) t_{i}}+\frac{h}{2}\left[\widetilde{A}\left(t_{0}\right)\left(\tilde{Q}^{\prime}\left(t_{i}-t_{0}\right)\right)+2 \sum_{k=1}^{i-1} \widetilde{A}\left(t_{k}\right)\left(\tilde{Q}^{\prime}\left(t_{i}-t_{k}\right)\right)+\widetilde{A}\left(t_{i}\right)\left(\tilde{Q}^{\prime}(0)\right)\right]
$$

Therefore,

$$
\begin{aligned}
\left|A\left(t_{i}\right)-\widetilde{A}\left(t_{i}\right)\right| \leq & \frac{h}{2}\left[\left|A\left(t_{0}\right)-\widetilde{A}\left(t_{0}\right)\right|\left|\tilde{Q}^{\prime}\left(t_{i}-t_{0}\right)\right|+2 \sum_{k=1}^{i-1}\left|A\left(t_{k}\right)-\widetilde{A}\left(t_{k}\right)\right|\left|\tilde{Q}^{\prime}\left(t_{i}-t_{k}\right)\right|\right. \\
& \left.+\left|A\left(t_{i}\right)-\widetilde{A}\left(t_{i}\right)\right|\left|\tilde{Q}^{\prime}\left(t_{0}\right)\right|+\left|A\left(t_{0}\right)+2 \sum_{k=1}^{i-1} A\left(t_{k}\right)+A\left(t_{i}\right)\right|\left|O\left(h^{4}\right)\right|\right]+\left|O\left(h^{2}\right)\right| \\
\leq & h L \sum_{k=0}^{i}\left|A\left(t_{k}\right)-\widetilde{A}\left(t_{k}\right)\right|+T\left|O\left(h^{4}\right)\right|+\left|O\left(h^{2}\right)\right|,
\end{aligned}
$$

where $L=\max _{0 \leq k \leq n}\left|\widetilde{Q}^{\prime}\left(t_{k}\right)\right|$. Then based on Lemma 3, we can get

$$
\max _{0 \leq i \leq n}\left|A\left(t_{i}\right)-\widetilde{A}\left(t_{i}\right)\right| \leq H \mathrm{e}^{2 T L},
$$

where $H=T\left|O\left(h^{4}\right)\right|+\left|O\left(h^{2}\right)\right|$. It is obvious that when $h$ tends to be zero, the error tends to be zero.

\subsection{Numerical Simulation}

In this subsection, we will use two numerical examples to show the validity of our result and check whether there exist IA fluctuations for two-unit series system with three states.

Case 1. We assume the fault, delay-repair and repair rates are as follows:

$$
\begin{aligned}
& \lambda_{1}=1, \mu_{1}=1, v_{1}=1, \\
& \lambda_{2}=2, \mu_{2}=1, v_{2}=2 .
\end{aligned}
$$

Then following the steps in Section 2.2, we can obtain the IA shown in the Figures 2 and 3: 


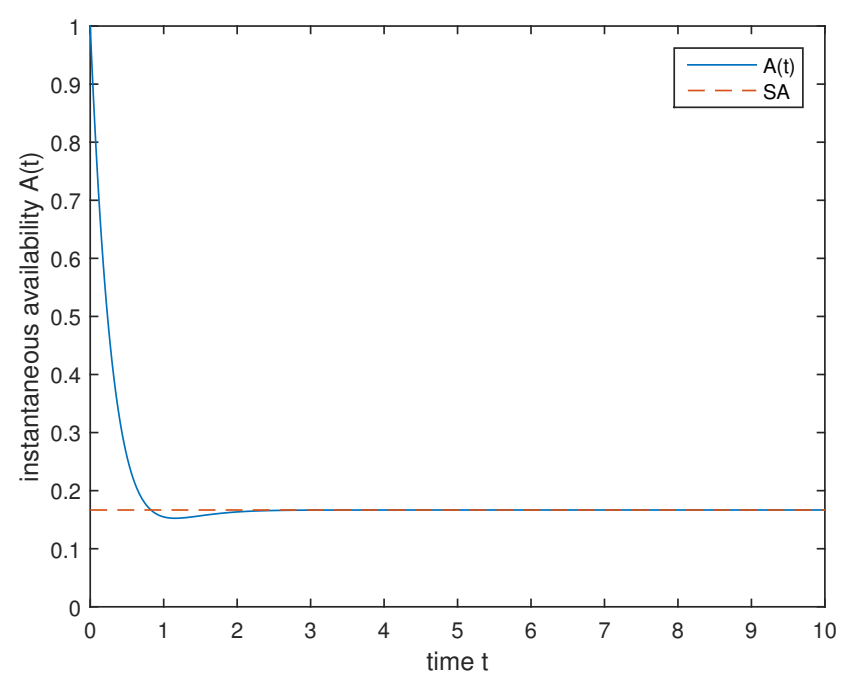

Figure 2. The curve of IA for case 1.

Case 2. We assume the fault, delay-repair and repair rates are as follows:

$$
\begin{aligned}
& \lambda_{1}=1, \mu_{1}=1, \nu_{1}=1 \\
& \lambda_{2}=2, \mu_{2}=5, v_{2}=2 .
\end{aligned}
$$

Then we can obtain the IA as follows:

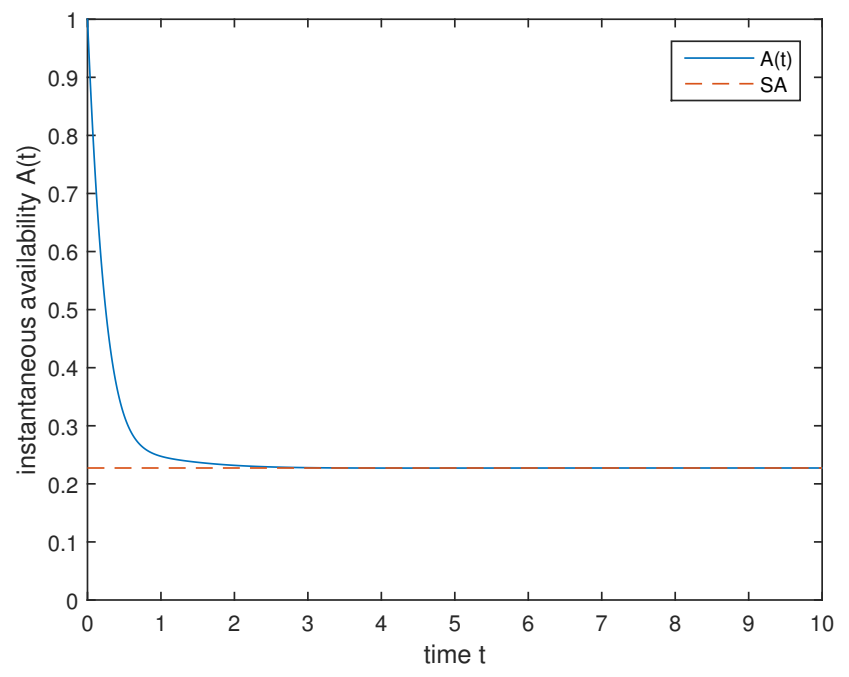

Figure 3. The curve of IA for case 2.

We can easily find from Figures 2 and 3 that there exists IA fluctuation in Case 1 but Case 2 does not. According to two cases, we can draw the conclusion that IA for the two-unit series system with three states has fluctuation under certain conditions.

\section{IA for Two-Unit Parallel System with Three States}

When the system is working, the unit cannot be repaired immediately when it is down, so there usually exists the delay-repair time. Three states (up, delay-repair and repair) is more realistic than two states(up and down). In this section, we will analyze the fluctuation of IA for two-unit parallel system with three states. 


\subsection{Mathematical Model of IA}

According to [22], for establishing the Markov model of IA for the two-unit parallel system with three states, the system must meet the following assumptions.

Assumption 3. Each unit has its own maintenance equipment, when the unit is down, its maintenance equipment will repair the unit immediately.

Assumption 4. Each unit $i$ has the working time $X_{i}$, the delay-repair time $W_{i}$ and repair time $Y_{i}(i=1,2)$, which are subject to the following exponential functions

$$
\begin{aligned}
X_{i} & \sim F_{i}(t)=1-e^{-\lambda_{i} t}, \\
W_{i} \sim W_{i}(t) & =1-e^{-v_{i} t}, \\
Y_{i} \sim G_{i}(t) & =1-e^{-\mu_{i} t},
\end{aligned}
$$

where $\lambda_{i}$ is the fault rate, $\mu_{i}$ delay-repair rate and $v_{i}$ repair rate.

Assumption 5. After maintenance of the unit, it will be as new and we suppose that at the beginning all units are new.

Under these assumptions, we can get all states of the system:

state 0: unit 1 and unit 2 are up.

state 1: unit 1 is up, unit 2 is delay-repair.

state 2: unit 1 is delay-repair, unit 2 is up.

state 3: unit 1 is up, unit 2 is repaired.

state 4: unit 1 is repaired, unit 2 is up.

state 5: unit 1 is delay-repair, unit 2 is delay-repair.

state 6: unit 1 is delay-repair, unit 2 is repaired.

state 7: unit 1 is repaired, unit 2 is delay-repair.

state 8: unit 1 is repaired, unit 2 is repaired.

The state transition diagram is shown in Figure 4.

According to [22], for this Markov process, we have the state transfer probability function as follows

$$
\left\{\begin{array}{l}
P_{00}(\Delta t)=1-\left(\lambda_{1}+\lambda_{2}\right) \Delta t+o(\Delta t), P_{01}(\Delta t)=\lambda_{2} \Delta t+o(\Delta t), P_{02}(\Delta t)=\lambda_{1} \Delta t+o(\Delta t), \\
P_{11}(\Delta t)=1-\left(\lambda_{1}+v_{2}\right) \Delta t+o(\Delta t), P_{13}(\Delta t)=v_{2} \Delta t+o(\Delta t), P_{15}(\Delta t)=\lambda_{1} \Delta t+o(\Delta t), \\
P_{22}(\Delta t)=1-\left(\lambda_{2}+v_{1}\right) \Delta t+o(\Delta t), P_{24}(\Delta t)=v_{1} \Delta t+o(\Delta t), P_{25}(\Delta t)=\lambda_{2} \Delta t+o(\Delta t), \\
P_{30}(\Delta t)=\mu_{2} \Delta t+o(\Delta t), P_{33}(\Delta t)=1-\left(\lambda_{1}+\mu_{2}\right) \Delta t+o(\Delta t), P_{36}(\Delta t)=\lambda_{1} \Delta t+o(\Delta t), \\
P_{40}(\Delta t)=\mu_{1} \Delta t+o(\Delta t), P_{44}(\Delta t)=1-\left(\lambda_{2}+\mu_{1}\right) \Delta t+o(\Delta t), P_{47}(\Delta t)=\lambda_{2} \Delta t+o(\Delta t), \\
P_{55}(\Delta t)=1-\left(v_{1}+v_{2}\right) \Delta t+o(\Delta t), P_{56}(\Delta t)=v_{2} \Delta t+o(\Delta t), P_{57}(\Delta t)=v_{1} \Delta t+o(\Delta t), \\
P_{62}(\Delta t)=u_{2} \Delta t+o(\Delta t), P_{66}(\Delta t)=1-\left(v_{1}+u_{2}\right) \Delta t+o(\Delta t), P_{68}(\Delta t)=v_{1} \Delta t+o(\Delta t), \\
P_{71}(\Delta t)=u_{1} \Delta t+o(\Delta t), P_{77}(\Delta t)=1-\left(v_{2}+u_{1}\right) \Delta t+o(\Delta t), P_{78}(\Delta t)=v_{2} \Delta t+o(\Delta t), \\
P_{83}(\Delta t)=u_{1} \Delta t+o(\Delta t), P_{84}(\Delta t)=u_{2} \Delta t+o(\Delta t), P_{88}(\Delta t)=1-\left(u_{1}+u_{2}\right) \Delta t+o(\Delta t),
\end{array}\right.
$$

and the elements $m_{i j}$ of the transfer rate matrix $M$ satisfies

$$
\left\{\begin{array}{l}
\lim _{\Delta t \rightarrow 0} \frac{P_{i j}(\Delta t)}{\Delta t}=m_{i j} \quad i \neq j, \\
\lim _{\Delta t \rightarrow 0}-\frac{1-P_{i i}(\Delta t)}{\Delta t}=m_{i i} .
\end{array}\right.
$$


So the transfer rate matrix $M$ is as follows

$$
M=-\left[\begin{array}{ccccccccc}
\lambda_{1} & -\lambda_{2} & -\lambda_{1} & 0 & 0 & 0 & 0 & 0 & 0 \\
0 & \lambda_{1}+v_{2} & 0 & -v_{2} & 0 & -\lambda_{1} & 0 & 0 & 0 \\
0 & 0 & \lambda_{2}+v_{1} & 0 & -v_{1} & -\lambda_{2} & 0 & 0 & 0 \\
-u_{2} & 0 & 0 & \lambda_{1}+u_{2} & 0 & 0 & -\lambda_{1} & 0 & 0 \\
-u_{1} & 0 & 0 & 0 & \lambda_{2}+u_{1} & 0 & 0 & -\lambda_{2} & 0 \\
0 & 0 & 0 & 0 & 0 & v_{1}+v_{2} & -v_{2} & -v_{1} & 0 \\
0 & 0 & -u_{2} & 0 & 0 & 0 & v_{1}+u_{2} & 0 & -v_{1} \\
0 & -u_{1} & 0 & 0 & 0 & 0 & 0 & v_{+} u_{1} & -v_{2} \\
0 & 0 & 0 & -u_{1} & -u_{2} & 0 & 0 & 0 & u_{1}+u_{2}
\end{array}\right]
$$

Let $P(t)=\left(P_{0}(t), P_{1}(t), P_{2}(t), P_{3}(t), P_{4}(t), P_{5}(t), P_{6}(t), P_{7}(t), P_{8}(t)\right)$, where $P_{i}(\mathrm{t}), i=0,1,2,3$, denote the probability that state $i$ occur at time $t$. So the ordinary differential equation of $P(t)$ can be obtained as follows

$$
\left\{\begin{array}{l}
P^{\prime}(t)=P(t) M \\
P(0)=(1,0,0,0,0,0,0,0,0)
\end{array}\right.
$$

For the parallel system, when the system is in state $E(E=0,1,2,3,4)$, the system is up. Combined with (35), if we can obtain $P(t)$, then we have $A(t)$ as

$$
A(t)=P_{0}(t)+P_{1}(t)+P_{2}(t)+P_{3}(t)+P_{4}(t) .
$$

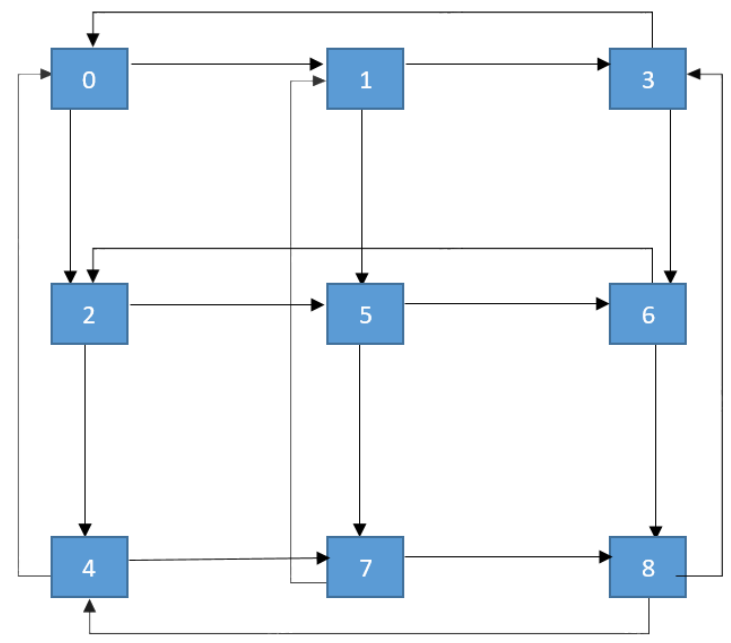

Figure 4. The state transition diagram of two-unit parallel system with three states.

\subsection{Numerical Solution and Error Analysis}

Because the dimension of the matrix $M$ is large, it is difficult to get the analytic solutions of IA. Therefore we will calculate out the numerical solution of $A(t)$. Next we will use four-order Runge-Kutta formula to get IA for the two-unit parallel system with three states.

Lemma 4 ([20]). For the ordinary differential equation with initial value

$$
\left\{\begin{array}{c}
\frac{d y}{d x}=f(x, y), \\
y\left(x_{0}\right)=a,
\end{array}\right.
$$


Four-order Runge-Kutta formula is as follows

$$
\left\{\begin{array}{l}
y_{n+1}=y_{n}+\frac{1}{6} h\left(k_{1}+2 k_{2}+2 k_{3}+k_{4}\right), \\
k_{1}=f\left(x_{n}, y_{n}\right) \\
k_{2}=f\left(x_{n}+\frac{1}{2} h, y_{n}+\frac{1}{2} h k_{1}\right) \\
k_{3}=f\left(x_{n}+\frac{1}{2} h, y_{n}+\frac{1}{2} h k_{2}\right) \\
k_{4}=f\left(x_{n}+h, y_{n}+h k_{3}\right) .
\end{array}\right.
$$

The error of four-order Runge-Kutta formula is $O\left(h^{5}\right)$.

Now we will apply four-order Runge-Kutta formula to (35) to get $A(t)$. According to Lemma 4, we have

$$
\left\{\begin{array}{l}
P\left(t_{i+1}\right)=P\left(t_{i}\right)+\frac{1}{6} h\left(k_{1}+2 k_{2}+2 k_{3}+k_{4}\right) \\
k_{1}=\left(P\left(t_{i}\right)\right) M \\
k_{2}=\left(P\left(t_{i}\right)+\frac{1}{2} h k_{1}\right) M \\
k_{3}=\left(P\left(t_{i}\right)+\frac{1}{2} h k_{2}\right) M \\
k_{4}=\left(P\left(t_{i}\right)+h k_{3}\right) M
\end{array}\right.
$$

Therefore $P\left(t_{i}\right)$ can be obtained, so that numerical solution of $A(t)$ can be gotten as follows:

$$
A\left(t_{i}\right)=P_{0}\left(t_{i}\right)+P_{1}\left(t_{i}\right)+P_{2}\left(t_{i}\right)+P_{3}\left(t_{i}\right)+P_{4}\left(t_{i}\right),
$$

and the error of $A(t)$ is $O\left(h^{5}\right)$.

\subsection{Numerical Simulation}

In this subsection, we will use two numerical examples to show the validity of our result and check whether there exist IA fluctuations for two-unit parallel system with three states.

Case 3. We assume the fault, delay-repair and repair rates are as follows:

$$
\begin{aligned}
& \lambda_{1}=1, \mu_{1}=1, v_{1}=1, \\
& \lambda_{2}=1, \mu_{2}=1, v_{2}=1 .
\end{aligned}
$$

Then following the steps in Section 3.2, we can obtain the IA shown in the Figures 5 and 6: 


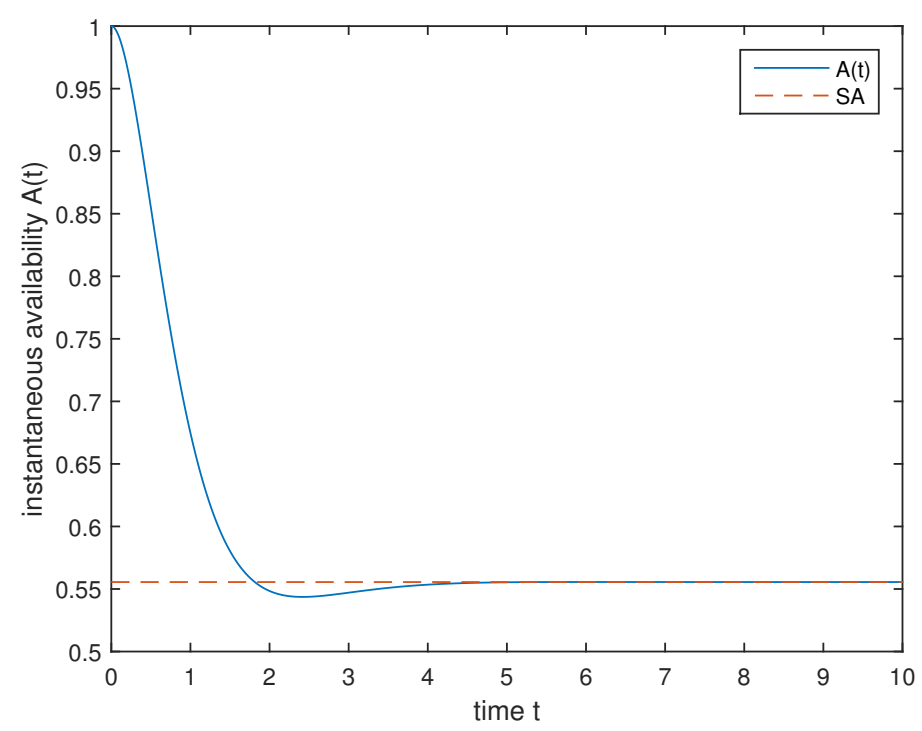

Figure 5. The curve of IA for case 3.

Case 4. We assume the fault, delay-repair and repair rates are as follows:

$$
\begin{aligned}
& \lambda_{1}=0.1, \mu_{1}=0.1, v_{1}=1, \\
& \lambda_{2}=1, \mu_{2}=1, v_{2}=1 .
\end{aligned}
$$

Then we can obtain the IA as follows:

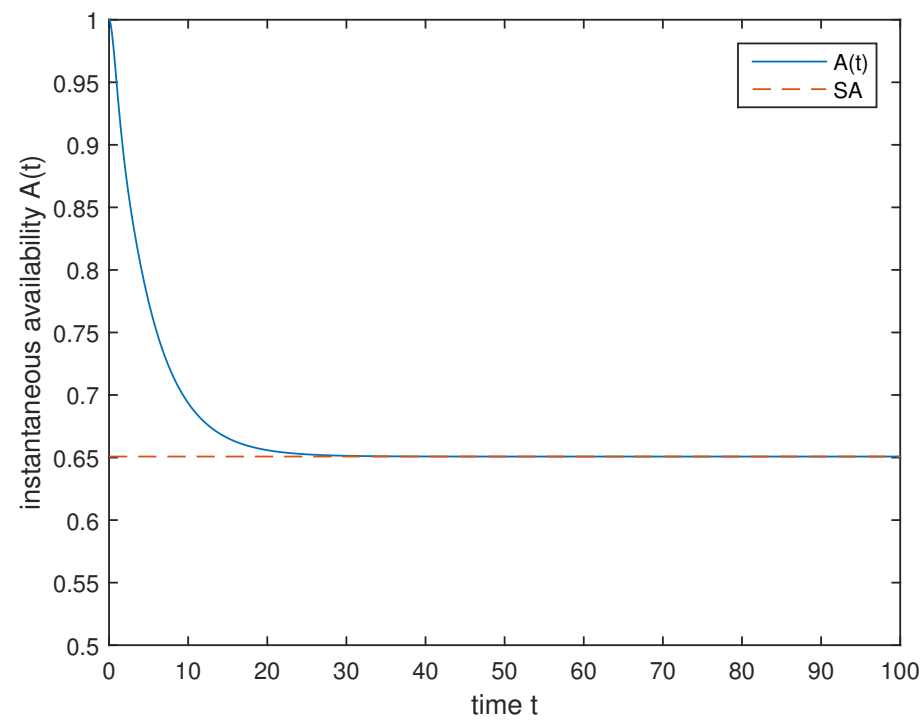

Figure 6. The curve of IA for case 4 .

We can easily find from Figures 5 and 6 that there exists IA fluctuation in Case 3 but Case 4 does not. According to two cases, we can draw the conclusion that IA for the two-unit parallel system with three states has IA fluctuation under certain conditions.

\section{Conclusions}

In this paper, we have present how to set up an IA model, solve the IA and analyze the IA fluctuation for two-unit series repairable systems and parallel repairable systems with three states. Firstly, based on the renewal process, we get the numerical expression of IA for the two-unit series system with three states. Then based on the Markov process, the numerical solution of IA for the 
two-unit parallel system with three states is obtained. The numerical simulation shows that there exist IA fluctuations for both two-unit series and parallel system under certain conditions. However, the reason why there exist IA fluctuations for two-unit series and parallel system with three states and how to reduce or eliminate fluctuations are still unclear. An in-depth study of the fluctuation mechanism is needed and we are focusing on it. Moreover, the IAs for systems under non-exponential distribution have not been studied. These problems will be studied in our future papers.

Author Contributions: Conceptualization, Y.Y.; Methodology, Q.L.; Software, Q.L.; Validation, Y.Y., Q.L. and X.C.; Formal Analysis, Q.L.; Investigation, Y.Y.; Resources, Y.Y.; Data Curation, Q.L.; Writing-Original Draft Preparation, Q.L.; Writing-Review and Editing, X.C.; Visualization, X.C.; Supervision, X.C.; Project Administration, Y.Y.; Funding Acquisition, Y.Y.

Funding: This research was funded by the (National Natural Science Foundation of China) grant number (61573041, 71671009).

Conflicts of Interest: The authors declare no conflicts of interest.

\section{References}

1. Wang, K.; Liu, Y.; Yin, M.; Jiang, H. Analysis on the instantaneous availability of series repairable systems in cyber warfare environment. In Proceedings of the 33rd Chinese Control Conference, Nanjing, China, 28-30 July 2014. [CrossRef]

2. Li, J.; Teng, K.; Yang, C.; Li, B. Research on the SMTN-based instantaneous availability of military aircraft in the mission preparation period. In Proceedings of the 2016 7th International Conference on Mechanical and Aerospace Engineering (ICMAE), London, UK, 18-20 July 2016. [CrossRef]

3. Siergiejczyk, M. Reliability Model of Motorway Emergency Communication System. Solid State Phenom. 2013, 210, 122-129. [CrossRef]

4. Xue, A.; Jing, Q.; Luo, L.; Wang, J.; Wang, B.; Huang, S.; Bi, T. Instantaneous availability for the protection devices based on non-homogeneous Markov model and a case study. In Proceedings of the 33rd Chinese Control Conference, Nanjing, China, 28-30 July 2014. [CrossRef]

5. Li, X.; Yang, Z.; Li, G.; Zhao, H.; Chen, F.; Zhu, X. Instantaneous availability modelling of CNC machine tool based on functional data analysis. J. Jilin Univ. (Eng. Technol. Ed.) 2012, 42, 1151-1156.

6. Sericola, B. Interval-availability distribution of 2-state systems with exponential failures and phase-type repairs. IEEE Trans. Reliab. 1994, 43, 335-343. [CrossRef]

7. Raje, D.; Olaniya, R.; Wakhare, P.; Deshpande, A. Availability assessment of a two-unit stand-by pumping system. Reliab. Eng. Syst. Saf. 2000, 68, 269-274. [CrossRef]

8. Mi, J. Limiting availability of system with non-identical lifetime distributions and non-identical repair time distributions. Stat. Probab. Lett. 2006, 76, 729-736. [CrossRef]

9. Sun, H.; Han, J.J. Instantaneous availability and interval availability for systems with time-varying failure rate: Stair-step approximation. In Proceedings of the 2001 Pacific Rim International Symposium on Dependable Computing, Seoul, Korea, 17-19 December 2001. [CrossRef]

10. Yang, Y. General Probability Distribution System Instantaneous Availability Discrete Time Modeling Analysis and Application. Ph.D. Thesis, Nanjing University of Science and Technology, Nanjing, China, 2008.

11. Yang, Y.; Kang, R.; Yu, Y.; Wang, L. Comparative Analysis of the Instantaneous Availability for Continuous-time and Discrete-time model on the One-Unit Repairable System. J. Converg. Inf. Technol. 2012, 7, 258-266.

12. Yi, Y.; Lichao, W.; Rui, K. Comparative analysis of the continuous-time model and discrete-time instantaneous availability model of the delay repairable system. In Proceedings of the 2011 IEEE 3rd International Conference on Communication Software and Networks, Xi'an, China, 27-29 May 2011. [CrossRef]

13. Ren, S.; Yang, Y.; Xu, H. Fluctuation analysis of instantaneous availability under delay-repair. In Proceedings of the 2016 35th Chinese Control Conference (CCC), Chengdu, China, 27-29 July 2016. [CrossRef]

14. Sarkar, J.; Biswas, A. Availability of a one-unit system supported by several spares and repair facilities. J. Korean Stat. Soc. 2010, 39, 165-176. [CrossRef] 
15. Yang, Y.; Wang, L.; Kang, R. Construction and Simulation Analysis for Stability Speed Parameter of Instantaneous Availability for One-Unit Repairable Systems. In Intelligence Science and Big Data Engineering; Lecture Notes in Computer Science; Springer: Berlin/Heidelberg, Germany, 2013; pp. 835-843. [CrossRef]

16. Ren, S.; Yang, Y.; Chen, Y.; Kang, R. Fluctuation analysis of instantaneous availability under minor repair. J. Beijing Univ. Aeronaut. Astronaut. 2017, 43, 602-607.

17. Li, Q.; Yang, Y.; Chen, X.; Xu, H. Fluctuation analysis of instantaneous availability for the series repairable system. In Proceedings of the 36th Chinese Control Conference, Dalian, China, 26-28 July 2017; pp. 7443-7447.

18. Zhao, D.; Liu, S. Failure model analysis of communication system in intelligent substation. J. Jiyuan Vocat. Tech. Coll. 2014, 2, 9-12.

19. Hariga, M.A. A maintenance inspection model for a single machine with general failure distribution. Microelectron. Reliab. 1996, 36, 353-358. [CrossRef]

20. Guan, Z.; Lu, J. Numerical Analysis; Higher Education Press: Beijing, China, 2010.

21. Lv, T.; Huang, J. High Precision Algorithm for Integral Equation; Science Press: Beijing, China, 2013.

22. Cao, J.; Cheng, K. A Mathematical Introduction to Reliability; Higher Education Press: Beijing, China, 2012.

(C) 2018 by the authors. Licensee MDPI, Basel, Switzerland. This article is an open access article distributed under the terms and conditions of the Creative Commons Attribution (CC BY) license (http:/ / creativecommons.org/licenses/by/4.0/). 